\title{
Zusammenhalt und Differenz
}

\section{Perspektiven auf Soziale Arbeit in Brasilien, oder: Zum Umgang mit Heterogenität dort, wo das Soziale extrem ungleich ist}

\author{
Benjamin Bunk • Emil A. Sobottka
}

Eingegangen: 10. September 2020 / Angenommen: 15. Oktober 2020 / Online publiziert: 4. Dezember 2020

(C) Der/die Autor(en) 2020

Zusammenfassung Eine wesentliche Debatte in Deutschland dreht sich um das Spannungsverhältnis von Zusammenhalt und Differenz, wie auch der Umgang mit Heterogenität seit jeher konstitutiv für die Sozialpädagogik ist. Vor dem Hintergrund von feinen Vereinnahmungen und Verschiebungen des sozialen Zusammenhaltes hier, wirft der Beitrag zunächst einen gesellschaftstheoretischen Blick auf die historisch wie aktuell umkämpfte Verfassung des Sozialen in Brasilien. Dort, wo das Soziale extrem ungleich ist und grobe Unterschiede herrschen, haben sich Ansätze Sozialer Arbeit herausgebildet, die sich der guten Bewältigung des sozialen Zusammenhaltes verschrieben haben, aber diesen von Erfahrungen der Differenz aus bearbeiten. Gerade weil sie individuelle Bildungsprozesse im Ringen um eine einseitig aufgekündigte Idee des Sozialen im Blick haben, zielen sie auf die Stärkung kollektiver Bewegungen, lokaler Wissenskulturen und alternativer (tradierter) Lebensweisen im urbanen wie im ländlichen Raum. Der Beitrag wendet daher den Blick, um anhand der versammelten Beiträge dieses Themenheftes vom Globalen Süden zu lernen sowie Brücken zur hiesigen Diskussion herauszuarbeiten. Sozialpädagogische Denkfiguren und postkoloniale Theorieansätze werden anhand der Frage verwickelt, wie man von einer Kritik der Verhältnisse zur guten Gestaltung von Praxis gelangt.

Schlüsselwörter Postkolonialismus · Partizipation · Soziale Ungleichheit · Gesellschaftstheorie $\cdot$ Sozialpädagogik

\footnotetext{
Dr. B. Bunk $(\varangle)$

Pädagogik des Jugendalters/Institut für Erziehungswissenschaft, Justus-Liebig-Universität Gießen, Karl-Glöckner-Str. 21B, 35394 Gießen, Deutschland

E-Mail: benjamin.bunk@erziehung.uni-giessen.de

Prof. Dr. E. A. Sobottka

Escola de Humanidades/Sociology, Pontifícia Universidade Católica do Rio Grande do Sul, Av. Ipiranga, 6681 - Partenon, 90619900 Porto Alegre, RS, Brasilien

E-Mail: esobottka@pucrs.br
} 


\section{Solidarity and difference}

Perspectives on social work in Brazil, or: how to deal with heterogeneity where society is extremely inequal

Abstract A major public debate in Germany revolves around the tension between solidarity and difference. Furthermore, the question of 'how to deal with heterogeneity' or, respectively, 'how to promote social cohesion', has always been constitutive for social pedagogy. Considering subtle appropriations and shifts within 'the social' here, the article first looks at the historically and currently contested constitution of society in Brazil. There, where social inequality is extreme and gross differences rule, approaches to social work have emerged which are committed to social cohesion, but are based on experiences of difference. As they focus on the political constitution of subjectivities within and through struggles for a (one-sidedly abandoned) idea of the social, they aim to strengthen collective movements, local knowledge cultures, and alternative (traditional) ways of life in urban and rural areas. Thus, on the one hand, the article turns the view towards the Global South, in order to learn from the subsequent contributions in this thematic issue. On the other hand, it seeks to build bridges towards the German discourse, intertwining socialwork thoughts and postcolonial theories of difference to answer the question of how to move from social critique to good practice.

Keywords Postcolonialism · Participation · Social inequality · Social theory · Social cohesion

\section{Zusammenhalt und Differenz oder: Vom Globalen Süden lernen?}

Dieser Blickpunkt möchte das spannungsreiche Verhältnis von Zusammenhalt und Differenz für die Soziale Arbeit wieder stärker in den Fokus rücken, verändert sich dieses doch aktuell. Provoziert durch die jüngeren Eindrücke von Spaltung, polarisierten Blasen, dem Erstarken rechter Positionen und populistischen Identitätspolitiken (auch hierzulande, aber noch viel deutlicher andernorts), wird als Reaktion darauf in Deutschland zunehmend der gesellschaftliche Zusammenhalt programmatisch. Gerade aufgrund der deutschen Geschichte(n) liegt dies auch zu Recht nahe. Doch die Betonung des Gemeinsamen sowie die Forderung nach der Notwendigkeit des Dialogs mit den Anderen, vereinnahmt und verdrängt zugleich die Position der anderen Anderen: der Stimmlosen hier, den global Ausgeschlossenen dort, wie übrigens auch der künftigen Generation. Insbesondere dort, wo die Rede vom Zusammenhalt mit normativem Impetus vorgetragen wird, wird sie gegenüber der Idee der zuhörenden Anerkennung von Differenz in hegemonialer Weise wirkmächtig. Nur auf den ersten Blick ist die selbstgewiss vorgetragene Positionierung für Zusammenhalt und gegen Differenz mit einer egalitären oder sozialen Orientierung gleichzusetzen.

Dieses ambivalente Spannungsverhältnis von Zusammenhalt und Differenz ist für die Soziale Arbeit konstitutiv, weshalb uns eine Diskussion über den Umgang mit Heterogenität und die Förderung sozialen Zusammenhaltes notwendig scheint. 
Denn der „Umgang mit Normalität“ (Winkler 2006) und die beständige Auseinandersetzung damit, wie „das Soziale“ zu denken sei (u.a. Evers und Nowotny 1987; Lessenich 2008) gehören zu den Grundfragen der Sozialpädagogik. Sowohl das disziplinäre Selbstverständnis wie auch die konkrete pädagogische Gestaltung müssen daher immer wieder, zu jeder Zeit und an jedem Ort, verhandelt werden, um öffentliche Verschiebungen dieses Verhältnisses von Zusammenhalt und Differenz zu reflektieren.

$\mathrm{Zu}$ einem erneuerten Blick auf Zusammenhalt und Differenz wollen wir aber nicht dadurch anregen, dass wir die sozialpolitische Umfänglichkeit oder philosophische Tiefe dieses Zusammenhangs vollends ausloten, sondern indem wir uns kontrastivvergleichend mit Sozialer Arbeit in Brasilien befassen. Anhand der in diesem Heft versammelten, praxisbezogenen Beiträge aus Brasilien soll ein gemeinsames Paradigma im Umgang mit Heterogenität thematisch werden, welches sich dort etabliert hat, wo das Soziale extrem ungleich ist. Mehr noch ist in Brasilien doch weitaus grundlegender die Form der Gesellschaft selbst umkämpft, also das, was als soziale Einheit zu betrachten ist, oder wer - aus Sicht von wem - daran teilhaben darf. Insofern haben sich alle diese Ansätze Sozialer Arbeit (im weiteren Sinne) der guten Bewältigung des Sozialen verschrieben, bearbeiten dieses aber ausgehend von einer Perspektive der Differenz.

Unabhängig davon, ob man zunehmende Ungleichheit nun als eine globale Entwicklungstendenz betrachtet, die uns noch bevorsteht (Alvaredo et al. 2018), ${ }^{1}$ oder ob es eher eine Frage der zunehmenden Wahrnehmung der Andersheit von Lebenslagen ist, welche sich im nationalen (u.a. El-Mafaalani 2018) ebenso wie im globalen Bezugsrahmen (u. a. Lessenich 2016) erst noch durchsetzen muss, so lässt sich vom Globalen Süden lernen. Jenseits von aufschlussreichen Beispielen Sozialer Arbeit aus Brasilien, die in je spezifischen Problemkonstellationen situiert sind, zeigen die nachfolgenden Beiträge exemplarisch neue Themenfelder, andere institutionelle Praktiken und alternative Denkfiguren zum Umgang mit Heterogenität auf. Vermittelt durch den distanzierenden Blick auf das Fremde sollen so die Spannungen dieses Zusammenhangs in den Vordergrund rücken.

Entsprechend werden in einem einleitenden Problemaufriss Beobachtungen öffentlicher Debatten vorangestellt, weshalb im hiesigen Kontext die aktuelle Rede von Zusammenhalt als ambivalent zu betrachten ist. Diesen feinen Unterschieden gegenüber wird in den brasilianischen Kontext eingeführt, welcher sich durch grobe Unterschiede auszeichnet, konkurrieren dort doch unterschiedliche politische Projekte darum, wie das Soziale verfasst sein sollte. Vor diesem Hintergrund werden

\footnotetext{
1 Der weltweite Ungleicheitsreport 2018 stellt für Europa fest, dass $10 \%$ der Einkommensbezieher über $37 \%$ des Einkommens verfügen, die obersten $1 \%$ sogar über $12 \%$. In Brasilien verfügen $10 \%$ über $55 \%$ des Einkommens bzw. die obersten $1 \%$ über $28 \%$ (ebd., S. 66f.). Bezieht man sich auf Vermögen statt Einkommen, ist die Ungleichheit noch drastischer. Während aber dieses Verhältnis in den Ländern des Globalen Südens seit den 1980ern stagniert, entwickelt sich die Ungleichheit in den westlichen Ländern des Globalen Nordens zunehmend in diese Richtung, wenn auch bspw. in Europa langsamer als in den USA. Auch im ländlichen Raum Brasiliens ist das Bewusstsein um diese krasse Ungleichheit sehr hoch. Bereits 1996 stellte der Atlas des Grundbesitzes des staatlichen Instituts für Kolonialismus und Agrarreform (INCRA 1996) fest, dass die 1,7\% größten Großgrundbesitzer über 43,7\% der landwirtschaftlich nutzbaren Fläche verfügen (respektive die obersten $10 \%$ über $80 \%$ der Fläche).
} 
dann unterschiedliche Konzeptionen Sozialer Arbeit, hier wie dort, auf der Suche nach Brücken und Orientierungen für die Gestaltung verwickelt.

\section{Problemaufriss: zur öffentlich verhandelten Ambivalenz von Zusammenhalt oder Differenz}

Diesen Sommer hat Black Lives Matter eine neue Generation berührt. Auch jenseits der USA, ihrer gegenwärtigen politischen Situation und der unmittelbar durch Sklaverei geprägten Geschichte, sind junge Menschen in Deutschland gegen strukturellen Rassismus auf die Straßen gegangen. Deren Empörung und Solidarität für Betroffene, mitunter Opfer, ist bemerkenswert. Ohne auf dieselbe Geschichte blicken zu können (in der die nichtweiße Haut oft ein Monument für rassistischen Missbrauch in der Vergangenheit ist), und auch ohne persönliche Ausgrenzungserfahrungen eint große Teile dieser (globalen) Generation offensichtlich die Überzeugung, dass Rassismus Unrecht ist - immer noch, wieder und in neu wahrnehmbaren Formen. Gewiss baut solch ein Bewusstsein hierzulande auf der kollektiven Erinnerung an andere historische Erfahrungen mit Rassismus auf, ebenso geht mit den sozialen Medien eine eindrückliche und ortsunabhängige Unmittelbarkeit aktueller Ereignisse einher. Dennoch scheint diese Generation angesichts einer allerorts spürbaren Renationalisierung und der generationalen Situierung in damit einhergehenden Politisierungsprozessen besonders aufmerksam für solche Ereignisse zu sein.

\subsection{Vereinnahmungen: Gleichheit oder Vielfalt}

Insofern verwunderte es (vielleicht), als aus feministischen, schwarzen oder migrantischen Diskursecken die Frage aufgeworfen wurde, ob diese symbolische Solidarität nun eigentlich gut ist oder nur gut gemeint (u.a. Della 2020). Der Vorwurf lautet, dass aus Sicht der eigentlich Betroffenen diese Solidarität ihre subalternen Stimmen in neuer Form vereinnahmt. Eine solche - letztlich selbstwertdienliche normative Positionierung lenke davon ab, dass die eigene Lebensweise, mitunter das bloße Weißsein, im Alltag Strukturen rassistischer Ausgrenzung reproduziert. Hörbar wurde dieses Dilemma beispielsweise dort, wo \#alllivesmatter getwittert, also der Verweis auf die Gleichheit der Menschenwürde betont wurde, was wiederum \#blacklivesmatter auf teils heftigste Weise verurteilte, als Missachtung von relevanter Verschiedenheit. Just aufgrund ihrer (äußerlich sichtbaren) Differenz sind jene Opfer von rassistisch motivierter Gewalt, wir anderen aber nicht. Oder, unter Verwendung einer Chiffre der Frauenbewegung aus den 1970ern: Who speaks for whom?

Wichtig ist dabei nachzuvollziehen, dass sich Formen der Gleichsetzung dahingehend unterscheiden, ob die für die Forderung nach Gleichberechtigung relevante Heterogenitätsdimension betont oder unterschlagen wird. Das Dilemma daran wird in einem anderen Zusammenhang deutlich: etwa dort, wo sich in Deutschland eine breite Front zivilgesellschaftlicher Kräfte gegen die fremdenfeindliche Haltung der AfD manifestiert (etwa auf \#Unteilbar-Demonstrationen). So ungemein wichtig es ist, lautstark eine Mehrheit für eine offene, demokratische Gesellschaft gegenüber 
ihren Gegner*innen zu artikulieren, so werden dennoch diejenigen anderen Anderen, die tatsächlich angefeindet oder abgeschoben werden, zugleich aufgrund der bereits bestehenden strukturellen Ausgrenzung noch leiser. Was sich dann beispielsweise in TV-Talkrunden zu Black Lives Matter spiegelte, in denen zwar offen über Rassismus diskutiert wurde, aber ohne Beteiligung einer Person of Color (PoC) oder einer Person mit Migrationsvordergrund. Jeweils sind die Adressat*innen, aber auch der Bezugsrahmen entscheidend dafür, welche Heterogenitätsdimension für eine Bewertung zum Tragen kommt. Also eher: Who speaks to whom? Ähnlich schildert beispielsweise Olivia Wenzel (2020) in dem Roman „1000 Serpentinen Angst“ zwar das persönliche Erleben von mehrfacher Benachteiligung als Schwarze, Ostdeutsche und Frau - muss sich aber auch eingestehen, dass sie im Hinblick auf andere Formen der Ungleichheit durchaus privilegiert ist, bspw. als Akademikerin aus der Mittelschicht im Globalen Norden. Ein Dilemma, das sich auch jenseits der individuellen Ebene spiegelt. Etwa dort, wo die Betonung auf dem ,,anthropogenen (menschengemachten) Klimawandel“ liegt, als Strategie zunächst gegenüber den Leugner*innen, vor allem aber, um die persönliche Verantwortung aller anzusprechen. Zugleich wird dadurch jedoch verdeckt, dass $70 \%$ der Menschheit zu arm sind, um hieran historisch einen Anteil zu haben, aber geografisch oder aufgrund ihrer sozial vulnerablen Position aktuell am stärksten betroffen sind (u. a. Gore et al. 2020; Moore 2016).

Bereits in den 1990er Jahren hatte Annedore Prengel (2007) darauf hingewiesen, dass Gleichheit und Vielfalt im Hinblick auf die pädagogische bzw. prozessorientierte Gestaltung ein komplementär-changierendes Spannungsfeld konstituieren (Prengel 2007, S. $51 \mathrm{ff}$.). So bedarf es einer Offenheit, gerade in der Moderation pädagogischer Settings, um den Freiraum für (unbekannte, letztlich grenzenlose) Vielfalt zu ermöglichen. Zugleich aber bedarf es einer gewissen (pädagogischen, hierarchischen) Struktur, damit Unterschiede in gleicher Weise gefördert werden können - und nicht nur die Lautesten sich durchsetzen oder jene Kompetenzen bedient werden, die aktuell en vogue sind. Sie macht in diesem Zusammenhang deutlich, dass es unterschiedliche „Heterogenitätsdimensionen“ gibt (Prengel 2007, S. 55) und auch das ,tertium comparationis“ (Prengel 2007, S. 56), die für alle verbindliche Vergleichsebene, für die Bewertung ob nun gerade Gleichheit oder Vielfalt $\mathrm{zu}$ fördern ist, entscheidend ist. Im Hinblick auf Migration hat jüngst Aladin ElMafaalani (2018) dieses Paradox von Offenheit und Struktur wieder prominent aufgegriffen, etwa dort, wo er - anschaulich anhand der Tischmetapher - Konflikte als Ausdruck gelingender Integration beschreibt (vgl. El-Mafaalani 2018, S. 76). So ermöglicht eine offene Gesellschaft zunehmend die (zuvor stimmlose) Artikulation von Differenz: Von Generation zu Generation trauen sich immer mehr Personen an den gedeckten Tisch, unzufrieden, mit den Resten abgespeist zu werden (wobei der analoge Verweis auf Geschlechtergerechtigkeit verdeutlicht, dass es eher um verdeckte, nicht um neue Konflikte geht). Dafür ist aber eine demokratische, alle umfassende sowie für alle verbindliche Grundstruktur notwendig, welche dieselben Teilhabechancen an der Gestaltung einer sich so verändernden Gesellschaft garantiert - in diesem Sinne ermöglichen Tischmanieren, dass neue Themen zur Sprache kommen oder andere Speisen serviert werden. 


\subsection{Verschiebungen: Allgemeines und Besonderes}

Die intentionale Gestaltung unserer Strukturen des Zusammenlebens, die geförderten Vergleichsebenen oder ermöglichten Bezugsrahmen, schlicht: der Umgang mit Heterogenität, ist aber selbst wieder Gegenstand von öffentlichen Auseinandersetzungen. Beispielsweise hat Saša Stanišić (2019) den deutschen Buchpreis erhalten. Bereits 2008 war er Adelbert-von-Chamisso-Preisträger, ein Preis für ,,auf Deutsch schreibende Autoren, deren Werk von einem Kulturwechsel geprägt ist“" und sich zugleich durch einen ,,außergewöhnliche[n], die deutsche Literatur bereichernde[n] Umgang mit Sprache“ auszeichnet ${ }^{2}$. Im Jahr 2017 wurde dieser weitsichtige „Preis zur Förderung von Migrationsliteratur“ dann eingestellt, da diese Autor*innengruppe in der deutschsprachigen Gegenwartsliteratur angekommen sei - was die Auszeichnung für Stanišić bestätigt. Oder?

Bereits 2014 kommentierte Maxim Biller diese Entwicklung in gewohnt bissiger Manier: Er warf der ,angepassten“ migrantischen Literatur einen „kalte(n), leeren Suhrkamp-Ton“ vor, wo Kultur „fast immer nur Folklore oder szenische Beilage“ sei, um damit das zu erfüllen, was „das harmoniesüchtige, postnazistische und vereinte Deutschland von ihnen noch mehr als von jedem seiner indigenen Künstler und Bürger erwartet" (Biller 2014). Seine migrantisch-postkolonial geschulte Beobachtung (die auch erheblich Widerspruch erfahren hat) war also, dass das Allgemeine nur vermeintlich heterogener wird, vielmehr hätte die Förderung von Differenz zur deren Anpassung verführt und nur die Regeln zum Ausschluss der anderen Anderen geändert. Als Intellektueller macht Biller (2019) dann auf eine ähnliche Entwicklung im Politischen aufmerksam (Biller 2019): Mit seiner Figur des Linksrechtsdeutschen reagiert er auf den neuen Duktus des „Wir müssen mit den Rechten reden“ gegenüber der Alternative für Deutschland (AfD) - und damit allen extremen Flügeln oder Positionen, etwa auch der Holocaust-Leugner*innen, innerhalb dieses Rahmens. Ein Duktus, der sich für ihn etwa im öffentlichen Rückgriff auf die persönlichen Seiten der gemeinsamen deutschen Geschichte (Biller 2019, etwa die Serie „Unsere Mütter, unsere Väter") oder in der Anerkennung von Rechten als diskursfähigen Intellektuellen in Zeitungen zeigt. In anderen Worten, hier werden bisherige Vergleichsebenen verschoben, welche den Dialog miteinander ermöglichen. Jetzt mag es für mich aus mehreren Gründen einsichtig sein, den Dialog mit Rechten zumindest zu führen. Aber dieser neue Dialog grenzt Jene aus (und darauf macht Biller in einem entscheidenden Satz aufmerksam), deren Verwandte im Holocaust vergast wurden oder die heute von Nazis angefeindet oder gar bedroht werden. Das spiegelt sich auch in öffentlichen Ereignissen: So hatte der Katholikentag in Münster (2018) die AfD explizit zum Dialog aufs Podium eingeladen - was zu Protesten, vor allem aber zum Fernbleiben vieler jüdischer Partner*innen führte, welche diese Grenze nicht mit überschreiten konnten. Der folgende evangelische Kirchentag (2019 in Dortmund) hatte daraus gelernt. Die entsprechende Erklärung sprach sich dann auch nicht gegen die Teilnahme von AfD-Mitgliedern aus, aber primär für den Dialog mit jenen anderen Anderen, ,die sich gegenwärtig in den gesellschaftlichen und politischen

\footnotetext{
2 Siehe „Adelbert-von-Chamisso-Preis der Robert Bosch Stiftung“: https://www.bosch-stiftung.de/de/ projekt/adelbert-von-chamisso-preis-der-robert-bosch-stiftung [Zugriff 20.10.2020].
} 
Debatten nicht wiederfinden"3. Dieser feine, aber sehr gravierende Unterschied in der Argumentation ging dann aber im Protestgetöse und dem linksrechtsdeutschen Vorwurf unter, eine Chance gegen Spaltung und für Zusammenhalt vertan zu haben.

Diese Ambivalenz im guten Umgang mit Heterogenität findet sich auch in einer aktuellen Kontroverse um die Ontologie kritischer Gesellschaftstheorie wieder (die sich wiederum auf die sachlogische Differenz von Situations- und Prozessorientierung in der Pädagogik beziehen lässt; was hier nicht vertieft werden kann). So gibt es jene Ansätze (geprägt bspw. durch Gender-, Queer- oder feministische Bewegungen), für die soziale Kämpfe, Antagonismen und Konflikte - die Differenz - den theoretischen Kern des Sozialen ausmachen. Wobei diese Antagonismen auch dem geschuldet sind, dass die Rede von der Gesellschaft oder dem Sozialen zunächst eine Totalität unterstellt, in der es sich dann durchzusetzen oder zu verteidigen gilt (Marchart 2010, 2019). Diese Theorielinie wird wiederum durch jene Traditionen kritisiert, in denen der Gestaltung des Gemeinwohls eine konstitutive Bedeutung zugeschrieben wird. So war etwa für Charles Taylor (2002) die Aushandlung politischer Positionen immer zugleich der Weg, um zwischen ausgeprägten Kultur- wie Sprachkreisen zu vermitteln. Darauf aufbauend, argumentiert jüngst Hartmut Rosa (2019) in pädagogischer Manier, dass die kollektive demokratische Aushandlung dessen, was als Kontingent und was als Basis (Totalität) betrachtet wird, für die individuellen Subjekte und ihre Anverwandlung von Welt konstitutiv ist und daher mehr auf die Möglichkeit der resonanten, sprich dynamisch-prozessorientierten Mitgestaltung ausgerichtet sein sollte und weniger auf die situativorientierte Repulsion der bestehenden Verhältnisse (vgl. Rosa 2019, S. 210).

\subsection{Relativierungen: Wahrheit und Position}

Solcherlei theoretische Überlegungen um umkämpfte Totalitäten und ihre Grenzen und - mehr noch - die öffentliche Forderung nach Zusammenhalt aus einem vagen Bedürfnis nach Stabilität und verlorener Eindeutigkeit heraus führen dazu, dass das Soziale im Sinne eines Gemeinwohls mitunter als Gegenüber von Diversität problematisiert wird: bedroht sowohl durch das Einfordern von stimmlosen oder Minderheitenpositionen (z. B. postkolonialen oder queeren Perspektiven) als auch durch die Infragestellung bestehender, jene Ausschlüsse produzierender Normierungen und Zuschreibungen (z.B. durch feministische Positionen). So liegt der Vorwurf in der Luft, dass auch eine linke Identitätspolitik wieder die hegemonialen Deutungsmuster diese Entwicklung der Spaltung und Renationalisierung vorbereitet hat (in Wochenzeitungen u.a. Newmark 2017; Flaßpöhler 2019; in feministischen Subkulturen bspw. Becker und Streiß 2018).

Einer der prominentesten Köpfe, welcher die unübersichtliche Kontingenz jeglicher Totalität vehement anprangert, ist Slavoj Žižek. In „Death of Truth“ (Žižek 2019, S. 103) vermisst er diese Debatte, indem er auf drei Entwicklungen hinweist. Zunächst setzt er den Aufstieg von religiösem Fundamentalismus einerseits und dem

\footnotetext{
3 Der originale „Doppelbeschluss von Dortmund“ vom Oktober 2018 ist online nicht (mehr) verfügbar, daher sei an dieser Stelle auf ein Interview mit dem damaligen Kirchentagspräsidenten Hans Leyendecker verwiesen (Leyendecker 2018).
} 
Fundamentalismus der politischen Korrektheit andererseits gleich (formal, aufgrund der strategischen Auswahl an Bezugsinformationen und der gezielten normativen Delegitimation des Anderen als nicht wahr). Zweitens spielen digitale Medien eine Rolle, in denen sich spezifische Überzeugungen finden, sich ihrer selbst vergewissern und radikalisieren. Drittens ist das Erbe der postmodernen Dekonstruktion und des historischen Relativismus die Einsicht, dass es trotz sorgfältigen wissenschaftlichen Argumentierens, seriöser Quellenauswahl und eines methodengeleiteten Erkenntnisprozesses keine objektive, allgemeingültige Wahrheit gibt. Diese beruht immer auf einem spezifischen Horizont, ist in einem subjektiven Standpunkt begründet und in gesellschaftlichen Machtverhältnissen verstrickt.

Vor diesem Hintergrund ordnet Žižek (2019) die Narrative neu, auch mittels einer bestimmten Sprachgebung (Žižek 2019, S. 104f.). Er beschreibt zum einen jene Position, die darauf pocht, dass Relativismus nicht ,anything goes“ bedeutet und dass es zwar Meinungen und Meinungsfreiheit gibt, aber eben auch härtere Fakten und stringentere Argumentationen. Diese käme mit einem unterschwelligen normativen Impetus der Aufrichtigkeit daher, der sowohl gegen links als auch rechts in Stellung gebracht wird, worin primär der Wunsch nach einem gemeinsamen Narrativ der Mitte, welches ,zusammenhält“, zum Ausdruck komme. Dieses habe es aber nur vermeintlich gegeben. Insofern ist - aus Sicht Žižeks - nicht der „Erfolg des postmodernen Relativismus“ “bzw. jene Identitätspolitik an den Rändern Schuld, sondern der Verfall der ,liberal-demokratischen Meistererzählung“, mit welcher das ,internationale Establishment" seine ideologische Hegemonie aufrechterhalten habe (Žižek 2019, S. 105). Demnach ist die Rhetorik von Zusammenhalt aus einer machtsensiblen Perspektive mehr als die Sehnsucht nach der Rückkehr zu einer weniger anstrengenden und weniger unsicheren Situation, in der man unhinterfragt mit der großen Wahrheit mitschwimmen konnte - wenn man denn auf der Sonnenseite dieser Meistererzählung geboren wurde. Oder etwa nicht?

Noch einmal: Wir changieren hier bewusst zwischen unterschiedlichen Positionen und Dimensionen, um der Ambivalenz dieses sich verändernden Zusammenhangs von Zusammenhalt und Differenz gerecht zu werden. Unser Anliegen ist nicht, einen bestimmten Standpunkt vorwegzunehmen. Aber die unhinterfragte Entscheidung für Zusammenhalt und gegen Differenz (oder andersherum) trägt vor dem aktuellen sozialpolitischen Horizont nicht (mehr) - noch mehr gilt dies für die Soziale Arbeit, die auszog, das Soziale gut zu gestalten. Und es ist eben diese vermeintliche Eindeutigkeit, die unter Bedingungen krasser sozialer Ungleichheit wie auch großer kultureller Diversität im Globalen Süden immer schon brüchig war. Darauf werden wir im nachfolgenden, zunächst gesellschaftstheoretisch orientierten und dann historisch-systematisch angelegten Unterkapitel zur (aktuell) umkämpften Verfassung des Sozialen in Brasilien eingehen.

\section{Gegenhorizont: die umkämpfte Verfassung des Sozialen in Brasilien}

Eine Debatte um Zusammenhalt setzt voraus, dass es etwas gibt, das zusammengehalten werden kann oder möglicherweise sogar soll. Schon auf die Frage, was dieses Etwas ist, gibt es je nach Kontext ganz unterschiedliche Antworten. Es kann zum 
Beispiel eine Gemeinschaft, eine Gesellschaft oder eine Nation sein, um nur drei sehr häufig vorausgesetzte Einheiten zu nennen, die auf einer gegenseitigen Bejahung der jeweiligen Teilnehmenden beruhen. Diese müssen eine affirmative Haltung zur Binnendifferenz einnehmen, erfahren aber angesichts dessen auch in subjektkonstituierender Weise ihre Differenz bzw. sich selbst als besonders und dennoch eingebettet. Der unsichtbare, aber doch als sehr real und legitim wahrgenommene Faden, der die Einheit zusammenhalten soll und den es gegebenenfalls zu schützen oder wiederherzustellen gilt, ist in jedem dieser drei genannten Beispiele ganz anderer Natur - worauf wir kurz eingehen wollen, bevor wir uns dem konkreten Fall Brasiliens zuwenden.

\subsection{Gesellschaftliche Einheit als soziales Konstrukt}

In seinem Aufruf für eine (umstrittene) Politik der Anerkennung der französischsprechenden Bevölkerung in Québec vertrat Charles Taylor (1994) vor einigen Jahren die Auffassung, dass eine Bevölkerung eine anzuerkennende und zu schätzende Wertegemeinschaft (in ethischer und kultureller Hinsicht) zu bilden habe und dass die (hier: französische) Sprache deren Zusammenhalt nach außen sichtbar mache (Taylor 1994). Anders als in älteren Auffassungen von der Einheit einer Gesellschaft, hat Taylor dabei nicht den Anspruch einer kompletten Anpassung (bzw. im Gegenzug den Ausschluss) aller in demselben Territorium lebenden Anderen erhoben, sondern versucht, eine von der Gemeinschaft einseitig definierte Toleranz hinsichtlich des Integrationszwangs für nur einen Teil der Anderen, nämlich der englischsprachigen Bevölkerung, zu begründen. Auf diese Auffassung von Zusammenhalt reagierte Jürgen Habermas (1994) mit der Verteidigung einer Idee des gesellschaftlichen Zusammenhalts, die sich auf politische Integration und Teilnahme als hinreichenden Ausdruck von Zugehörigkeit beschränkt. Dabei greift er auf seine These zurück, dass moderne Gesellschaften ihre Einheit durch die Verfassungsgebung als Rechtsgemeinschaft bilden. Diese Idee, die er als Verfassungspatriotismus beschreibt, fordert von ihren Mitgliedern die Einhaltung von Regeln, die zwar prinzipiell selbst (mit)bestimmt sind, als Rechtsform dann aber feststehen (Habermas 1992). Von neu dazukommenden Anwärter*innen auf eine Mitgliedschaft könne nicht die Aufgabe einer Lebensweise erwartet werden, die Ausdruck ihrer tiefen Verankerung in der jeweiligen Kultur sei. Eine dritte, sehr verbreitete Form, jene Einheit des Sozialen zu konzipieren, ist die der Nation. Als bindendes Element sind hier symbolische Zugehörigkeitsgefühle wirkmächtig. Der im wissenschaftlichen Alltag etablierte Begriff des ,nation-building“ verweist aber darauf, dass es sich entgegen ontologischen Wahrnehmungen eher um das Ergebnis von historischen Prozessen politischer Konstruktion handelt.

\subsection{Ursprünge einer hierarchischen Gesellschaft}

Erste Initiativen für lokale Nation-building-Prozesse ergriffen in Lateinamerika die oberen sozialen Schichten, um sich von den kolonialen Mutterländern (Spanien bzw. Portugal) abzugrenzen. Diese Vorhaben schlossen jedoch nie die gesamte lokale Be- 
völkerung ein ${ }^{4}$. Im besonderen Fall Brasiliens, das sich zunächst als unabhängiges Königreich behauptete, galt der erste Versuch, eine Einheit zu bilden, vorwiegend dem Aufbau einer Verwaltung. Erst ab 1870 im Zuge der positivistischen Bestrebungen zur Gründung einer Republik begann die Konstruktion einer symbolischen Einheit als „nação brasileira“ - zunächst vor allem in der Literatur und der politischen Presse (Oliveira 1990). Diese Nation sollte sich „,von einer rückständigen, theologisch-metaphysisch geprägten, zu einer fortschrittlichen positivistischen Gesellschaft" entwickeln, wobei in diesem Zusammenhang ,alle Nicht-Weißen“ als „Belastung für eine republikanische Entwicklung des Landes angesehen“ wurden (Sandkötter 1999, S. 123). Der öffentliche, offen abschätzige Diskurs gegenüber den Anderen - d. h. gegenüber den quilombolas, indígenas, mestiços sowie den armen, überwiegend ländlichen Bevölkerungsgruppen insgesamt - wandelte sich im Verlauf des 20. Jahrhunderts, insbesondere während der verschiedenen autoritären Regierungen (und Militärdiktaturen), zum gegenwärtigen Diskurs über die notwendige Modernisierung, deren Bewältigung jenseits der Anderen dann Zusammenhalt erfordert. Oder, wie Stephan Sandkötter dies zutreffend ausdrückt: „Die Heterogenität der verschiedenen Ethnien und auch Bevölkerungsschichten wird wegnationalisiert" (Sandkötter 1999, S. 130).

Eine solche Verfassung von Gesellschaft, bei begrenzter Inklusion von Teilen der Einwohner*innen, führt zu dem, was man als territoriale Integration (,integração territorial“) bezeichnen kann (Sobottka 2019). Die verschiedenen Bevölkerungsgruppen beziehen sich zwar auf dasselbe Territorium, also ihre Existenz auf einem bestimmten Gebiet, ohne aber eine bedeutungsreiche Einheit oder ein tragendes Bewusstsein wechselseitiger Zugehörigkeit auszubilden bzw. sich in anderen Dimensionen zu integrieren - geschweige denn, jenes kooperative Verhältnis auszubilden, dass Axel Honneth (2011) als „,soziale Freiheit“ beschreibt. Eine solche Gesellschaftsform, im Grunde ohne etablierte Heterogenitäts- wie Gleichheitsdimensionen, geht notwendigerweise mit gegenseitiger Entfremdung bei strikter Einhaltung und Akzeptanz der Hierarchie zwischen diesen Gruppen einher. Dieses Nebeneinander-Leben baut auf die Wahrung einer sozialen Distanz auf, jenseits derer sich Menschen nur aus funktionalen Gründen begegnen - und hält nur so lange, wie der Status quo nicht aktiv infrage gestellt bzw. mehr Inklusion gefordert wird. Etwa, wenn - mit gewissen Abstufungen - indígenas, quilombolas, Menschen in Favelas und Stadtrandgebieten, Migrant*innen, Frauen oder soziale Bewegungen der verarmten Landbevölkerung für mehr Teilhabe, Partizipation und Chancengleichheit aufbegehren - und dabei unterstützt werden, verstanden als Arbeit am Sozialen.

In jedem Fall gibt es zwischen lateinamerikanischen Ländern und einigen europäischen Ländern gravierende Unterschiede im Umgang mit Differenz bzw. der Art und Weise, wie die anderen als Andere für diejenigen in Erscheinung treten, und wer, nach eigener Auffassung, die Differenzierungsmerkmale sowie die Regeln ihrer möglichen Überwindung festlegen kann. Ein Grund dafür ist, dass es in Europa immer schon Ideen von Gesellschaft gab, welche jenseits der verschiedenen historischen Migrationsphänomene bemüht waren, einen Zusammenhalt trotz Differenz

\footnotetext{
${ }^{4}$ So wurde in Brasilien etwa der indigenen Bevölkerung erst mit der Verfassung von 1988 die brasilianische Staatsangehörigkeit zugeteilt.
} 
sicherzustellen - was nicht heißt, dass bspw. aktuelle Formen des Ausschlusses von Migrant*innen oder Asylsuchenden nicht vergleichbar mit Formen des Ausschlusses in Brasilien wären. Aber, um eine Metapher wieder aufzugreifen: In Lateinamerika haben sich selbsternannte Eliten im Festsaal an einen Tisch gesetzt und einen Teil der Bevölkerung als Sklav*innen, später Bedienstete bzw. Hausmädchen benutzt, ohne auch nur darüber nachzudenken, diese als würdig genug anzusehen, sich dazuzusetzen. Nicht am Katzentisch, sondern in der Küche durften diese sich von dem Übriggebliebenen ernähren. Die Mehrheit der Bevölkerung aber durfte sich dem Festmahl nicht nähern, ja nicht einmal das Blickfeld der geschlossenen Gesellschaft stören.

\subsection{Aktuelle Konstellation: Abbau oder Ausbau sozialer Ausgrenzungen?}

Wie oben bereits angedeutet, hat sich die Idee der Bildung einer brasilianischen Nation erst im späten 19. Jahrhundert konstituiert. Treffend analysiert etwa Lúcia Oliveira (1990), wie sich hernach drei konkurrierende Gesellschaftsprojekte herauskristallisierten, die von jeweils anderen sozialen Gruppen getragen wurden. In der dualistischen Gegenüberstellung von traditionell und modern aber hatten diese eine gemeinsame Diskursstrategie etabliert, die Diversität abschätzend als nachteilig und lästig darstellte ${ }^{5}$. Nun würde es hier zu weit führen, diese Entwicklung(en) und sukzessiven Verschiebungen nachzuzeichnen, aber: Der Konflikt um die grundlegende Gestalt der brasilianischen Gesellschaft ist bis heute nicht zufriedenstellend beigelegt. Ganz im Gegenteil: Acht Verfassungen seit 1891 (die Hälfte davon autoritär oktroyiert) und eine Vielzahl an gewalttätigen Machtübernahmen zeugen unmissverständlich von einem Kampf, nicht um Nuancen innerhalb einer zuvor gesetzten Einheit, sondern um die Macht über den Staatsapparat, mehr noch: die Gestaltung des Sozialen insgesamt. Auch gegenwärtig findet dieser Wettstreit der Gesellschaftsprojekte statt, ob nun bei dem parlamentarischen Putsch gegen die Arbeiterpartei und Präsidentin Dilma (2016), der Wahl Jair Bolsonaros (2018) oder aktuell um das Management der Corona-Pandemie. Unter dem Vorbehalt, dass bei jeder (knappen) Analyse eine gewisse perspektivische Zuspitzung erfolgt, sind für die politische Agenda aktuell maßgebend: ein sozialdemokratisches, ein autoritär-religiöses und ein wirtschaftsliberales Projekt.

Das sozialdemokratische Projekt hatte großen Einfluss auf die 1988 verkündete Verfassung. Es wurde erst später, insbesondere im Zuge des Widerstandes gegen die letzte Militärdiktatur (1964-1985), durch soziale Bewegungen, Gewerkschaften, Intellektuelle und aus Teilen des Mittelstandes sowie der katholischen und protestantischen Kirche heraus entwickelt. Die Vorstellungen und Erwartungen dieser konkreten Utopie bündeln sich in dem - an sich unpräzisen - Ausdruck ,cidadania (citizenship)“, während deren politische Vertretung sich über eine große Anzahl zivilgesellschaftlicher Vereinigungen und politischer Parteien zerstreut. Ihren am stärksten institutionalisierten Akteur fand dieses Projekt einer zunehmenden De-

\footnotetext{
5 Ein plastisches Beispiel ist das einflussreiche Buch „Os dois brasis“ („Die zwei Brasilien“) von Jacques Lambert, der 1957 den Ausdruck Belindia prägte: Brasilien als ein Land, das halb Belgien (modern) und halb Indien (traditionell, archaisch) ist.
} 
mokratisierung gewiss in der Arbeiterpartei (,,partido dos trabalhadores“; Sobottka 2006). In den Regierungsjahren (2003-2016) konnte diese Teile des Projektes umsetzen, etwa: erweiterte politische Partizipation, verstärkter Umweltschutz, eine Grundsicherung durch eine umfangreiche Sozialpolitik, der Ausbau eines allgemeinen Zugangs zu Bildung, Stärkung der Arbeitnehmerrechte, die Erhöhung des Mindestlohns oder die Anerkennung gleichgeschlechtlicher Ehen. Für die Etablierung einer offenen, demokratischen Gesellschaft war dies sicherlich ein signifikanter Anfang; die Gleichstellung und Durchmischung zuvor getrennter Gruppen sowie eine Umverteilung zwischen diesen waren spürbar. Aber es gab auch Grenzen. Die auf Großgrundbesitz basierende Agrarwirtschaft (siehe auch der Beitrag von M. Backhouse im Heft), das Rentseeking-System des gehobenen öffentlichen Dienstes oder die Dependenzstrukturen des Finanz- und Bankensystems konnten nicht angetastet werden, obgleich diese drei Bereiche zu den wichtigsten Ursachen der krassen sozialen Ungleichheit in Brasilien gehören.

Dennoch mobilisierte dieses kurze alternative Experiment aber auch eine unheilige Allianz des Widerstandes. So haben sich konservative Christen zunächst mit Neopfingstorganisationen ${ }^{6}$ gegen die Liberalisierung der Moral eingesetzt, während autoritäre Gruppierungen - die etwa für schärfere Strafen bei Verbrechen (inklusive der Todesstrafe) und eine grenzenlose Liberalisierung von Waffen eintreten einen (hasserfüllten) Diskurs zur Rettung vor dem Sozialismus etablierten. Symbolisch wurde in diesem Zuge öffentlich für die Wiederkehr des Militärregimes, die bürgerlich-konservative Familie, eine (privat wie öffentlich) religiös geleitete Lebensführung und Vaterlandsliebe plädiert. Im Sinne einer konkreten gesellschaftlichen Transformation gab es zuvor keine nennenswerten Gemeinsamkeiten zwischen diesen Gruppen; ihr Zusammenhalt konstituierte sich über den Widerstand gegen jene Personen, Institutionen und Gesetze, die für die Herstellung dieses sozialdemokratischen, auf Offenheit für Differenz bedachten Projekts einer umfassenden Idee des Sozialen standen. Offensichtlich wurde dieser Zusammenschluss 2016 durch den parlamentarischen Putsch gegen die Präsidentin Dilma Rousseff.

Ein drittes Projekt, das in Brasilien im Wettstreit um die politische Führung steht, wird vor allem vom Finanzmarkt, von Großunternehmern und Manager*innen sowie von zerstreuten Individuen getragen, die gemeinsam die wirtschaftliche Elite des Landes bilden. Der Fokus dieses Projektes liegt vorwiegend auf einer kurzfristigen Liberalisierung der Wirtschaft (Bresser-Pereira 2018), und seine sozialen Träger*innen plädieren für die Privatisierung von Staatsunternehmen und öffentlichen Diensten, die Abschaffung der umfangreichen Gesetzgebung zum Arbeiter*innen-, Umwelt- und Verbraucherschutz sowie eine drastische Reduzierung der Sozialpolitik. Die als notwendig angesehene Bekämpfung der absoluten Armut soll, im Sinne der Weltbank, in Form von Geldtransfers erfolgen; die Einheit aller wird so primär in der wirtschaftlichen Dimension verortet bzw. als Integration der Ausgeschlossenen in das Kreditsystem und in den Verbrauchermarkt verstanden. Andererseits

\footnotetext{
${ }^{6}$ Wir verwenden die Bezeichnung Organisation, weil es sich dabei überwiegend um gebündelte soziale Praktiken handelt, die ausdrücklich Dienste gegen Opfergaben tauschen. Sie bilden keine Gemeinden, die Mitglieder haben, sondern werben um ,customers“, obliegen aber auch keiner Rechenschaftspflicht gegenüber dem Staat.
} 
werden sehr umfangreiche staatliche Zuschüsse für private Investitionen und öffentliche Infrastruktur gefordert bei gleichzeitigen Steuersenkungen für Unternehmen und Finanzkapitalerträge. Kurzgefasst: ein minimaler Staat im Hinblick auf soziale oder kulturelle Belange, Bürger*innenrechte und demokratische Teilhabe, aber ein starker Staat als Verteidiger des allumfassenden Marktes und dessen Regelungsmechanismen.

Die aktuelle Regierung „Bolsonaro“ in Brasilien (2019-2022) wurde durch einen Schulterschluss der Träger des autoritär-religiösen und des wirtschaftsliberalen Projektes gebildet - was sich nicht nur im Diskurs der Regierung (u.a. Ghiringhelli de Azevedo 2020) in der Besetzung der Ministerien mit Militärangehörigen ${ }^{7}$ oder durch Kürzungen im Bereich Sozialer Arbeit zeigt (u.a. Estevam 2020). Während die Ersteren sich anstrengen, die ohnehin fragile soziale Sicherung und Sozialpolitik zurückbauen und eine exkludierende, religiös-national fundierte Symbolund Überwachungspolitik installieren, können gleichzeitig die Vertreter*innen des wirtschaftsliberalen Projektes relativ autonom die Wirtschafts- und Finanzpolitik, inklusive des öffentlichen Haushalts, bestimmen. Ein Ausdruck dessen, dass die Regierung ihre Politik darüber hinaus als Kulturkampf um das Soziale auffasst, ist beispielsweise die Universitätspolitik: Neben dem aktiven Eingreifen in Besetzungsverfahren von Rektor*innen, wurden die radikalen Kürzungen im Bereich der Sozial-, Geistes- und Kulturwissenschaften (u.a. die Streichung aller Stipendien) damit begründet, dass diese Bereiche nichts zur Produktivität der Gesellschaft beitrügen, sondern nur der Ausbildung sozialistischen Gedankenguts dienten. Aber auch bei deren Träger*innen (d.h. Wähler*innen) wurde das historische Motiv einer dualistischen Gesellschaftsform wirkmächtig. Aus deren Sicht gibt es zum einen die ,pessoas do bem“ (die Menschen des Guten), die zu ihrem elitär-exkludierenden Brasilien gehören, während allen Anderen die Zugehörigkeit verweigert wird - ein Brasilien, welches es dann vor all jenen Sozialist*innen zu verteidigen gilt, welche diese nicht-partizipative Vorstellung des Sozialen kritisieren oder aus einer ausgeschlossenen Position um Teilhabe ringen. Während die grüngelben Trikots der Fußballnationalmannschaft Brasiliens zuvor für die minimale, aber immerhin einende territoriale Integration als Nation standen, wurden sie durch dieses konservativnational-liberale Projekt vereinnahmt - und markieren inzwischen klar die Grenze der Zugehörigkeit zu den konkurrierenden Vorstellungen des Sozialen. Es wiederholt sich so eine Strategie aller autoritären Regierungen Brasiliens des 20. Jahrhunderts: Der emotionale Appell an nationale Symbole soll eine Einheit herstellen, welche die Verräter, die die Regierung nicht bedingungslos unterstützen, ausgrenzt.

\section{Verwicklung: das pädagogische Dilemma von (postkolonialer) Kritik und der Arbeit am Sozialen}

Ein minimaler Nenner Sozialer Arbeit in Brasilien und Deutschland ist die gerechte Bearbeitung sozialer Ungleichheit. Während für Profession wie Disziplin hierzulan-

\footnotetext{
7 Aktuell sind elf von 23 Ministerien durch Militärangehörige besetzt bzw. haben nur noch zwei Ministerien keine Militärs in hohen Positionen.
} 
de aber eher Begriffe wie „sozialer Zusammenhalt" wohl bedeutsam sind, ist die Debatte in Brasilien von extremer Ungleichheit geprägt - und damit durch existenzielle Armut, verwehrte Teilhabe, soziale Konflikte, politische Polarisierung und Klassenkämpfe gerahmt. Dies erklärt zum Teil, weshalb Theorien der Differenz, jüngst zunehmend unter das Label ,postkolonial“ subsumiert, für die Soziale Arbeit dort eine weitaus größere Rolle in Theorie und Praxis spielen. Neben der eigenen kolonialen Vergangenheit liegt dies auch gewiss daran, dass sich gegenwärtige Ungerechtigkeiten eines globalisierten Kapitalismus oder einer machtdurchdrungenen Realpolitik im Süden nicht so leicht ausblenden lassen wie im Norden (exemplarisch: Sousa Santos 1994; Lessenich 2016). Theorien der Differenz schwingen dort als Begründungsfiguren Sozialer Arbeit daher beständig mit, egal ob narrativ gerahmt als (gescheiterte) Befreiungsbewegung, (fortwährende) Dependenz oder Verwerfungen aufgrund wechselseitiger Zuschreibungen.

Gleichwohl ist das Anliegen dieses Abschnittes nicht, einen Überblick über Formen der Sozialarbeit in Brasilien vorzulegen, deren sozialgeschichtliche Genese zu erzählen oder eine (doch sehr anders disziplinär aufgespannte) Systematik zu entwerfen, sondern einen Dialog mit hiesigen Debatten zu ermöglichen. Das ist methodologisch insofern schwierig, als eine angemessene Vergleichsebene eben fehlt. Hier bestimmen staatliche Institutionen und soziale Sicherungssysteme, Sozialpolitik und Sozialrecht den Denk- und Handlungsrahmen der Sozialen Arbeit, was auch dazu führt, dass es ihr mitunter an theoretischen Grundlagen fehlt (vgl. Kessl 2011, S. 135). Indem sich Sozialarbeitsdiskurse aber auf die Prämissen existierender Institutionen und die bestehende soziale Ordnung stützen, sind sie nicht vergleichbar, und die hier gängigen Systematiken (etwa Otto et al. 2018) lassen sich nicht ohne Weiteres auf den Globalen Süden übertragen (zum Problem einer „Northern Theory“: Connell 2006; zum impliziten Nationalismus pädagogischer Erkenntnis: Mecheril et al. 2013, S. 7). Daher werden wir im folgenden Abschnitt Debatten der Sozialpädagogik hier wie der postkolonialen Theorie dort anhand eines gemeinsamen Problems in einen Dialog verwickeln: Wie kommt man von einer Kritik der bestehenden Verhältnisse zur Gestaltung guter Praxis?

\subsection{Konzeptionen des Sozialen}

Ein gängiges technisches Verständnis Sozialer Arbeit hier, das sich als sozialarbeitswissenschaftliches Paradigma (u. a. Staub-Bernasconi 2007) von einem sozialpädagogischen abgrenzt, baut einerseits auf einer Begründung von Sozialer Arbeit in Abgrenzung zu Familie und Schule auf (Bäumer 1929) und setzt andererseits einen Staat voraus, der die wohlfahrtsstaatliche Sorge jenseits dieser Einrichtungen übernimmt und der gesetzliche Regelungen für private Organisationen vorgibt oder Rechtsansprüche setzt, die es dann zu finanzieren gilt. Nicht eine bestimmte normative Orientierung ist also Ausgangspunkt für dieses Verständnis Sozialer Arbeit, sondern die empirisch gegebene Praxis.

\footnotetext{
8 FN/EN: Vgl. etwa die Definition der International Federation of Social Workers (IFSW) und der International Association of Schools of Social Work (IASSW) von 2014. Verfügbar u. a. unter: https://www.iasswaiets.org/de/global-definition-of-social-work-review-of-the-global-definition/ [Zugriff 20.10.2020].
} 
Möchte die Soziale Arbeit diesen institutionellen Rahmen verlassen - etwa, um eine mögliche Kritik des Vorgegeben formulieren zu können -, kommt sie nicht ohne ein Verständnis von Kultur aus, wie etwa Michael Winkler (2006) in seinem Entwurf einer Sozialpädagogik formuliert. Dieser etwa baut auf der Einsicht auf, dass unsere Gesellschaften nicht homogen, vielmehr unsere symbolischen Sinnzusammenhänge plural und kontingent sind und wir Individualität bzw. Subjektivität nicht ausblenden dürfen. Daher muss Soziale Arbeit gewissermaßen ausloten, ob und wie es Normalitätsfelder in unterschiedlicher Stärke und Verbindlichkeit gibt, sowie, welche sozial und kulturell zugestandenen Bandbreiten menschlichen Verhaltens existieren und wie die Grenzen der zu gestaltenden Lebensformen aussehen. Oder anders formuliert, kreist Sozialpädagogik ihrem Wesen nach um das Verhältnis von Zusammenhalt und Differenz. Eine Auffassung, so viel sei angemerkt, die dann aber voraussetzt, dass das Soziale als umfassend anerkannt wird, um nicht erst jenseits einer einseitig definierten Grenze zu Anderen Normalität auszuloten (siehe etwa Migrationspolitik).

Die postkoloniale Theoriebildung hingegen setzt keine Einheit des Sozialen voraus. Sie speist sich aus Erfahrungen der Exklusion und aus Kämpfen um Teilhabe und Anerkennung. Dabei ist ,postkolonial“" eher ein Sammelbegriff, der Konzepte auf unterschiedlichen Ebenen vereint. Im engeren Sinne ist damit ein Kern an kulturtheoretischen und dekonstruktivistischen Theorien gemeint (Said, Bhaba, Fannon, Hall, auch Spivak). Als Praxis der Kritik hatten diese nicht den Anspruch, konkrete Handlungsanweisungen (z. B. Soziale Arbeit) zu liefern. Insofern ist Postkolonialismus auf einer zweiten Ebene eine diskursive Strategie der Delegitimation des Bestehenden, um so Freiräume für die Artikulation subalterner Stimmen zu schaffen, aber auch, diese eigene Stimme anhand von Kämpfen um konkrete hegemoniale, essentialistische, stereotype oder disziplinierende Zuschreibungen auszubilden. Was auf einer dritten, praxisorientierten Ebene dann dazu führt, diverse, lokale, alternative, traditionelle Lebensweisen anzuerkennen und zu fördern und in Lateinamerika mit der Chiffre „,dekolonial“" markiert ist (Quijano 2000; Mignolo 2007) - und bisweilen zu einem (aus westlicher Perspektive) befremdlichen Stil zwischen polemisch zugrichtender Herrschaftskritik und emphatischer Darstellung des Anderen führt.

Insofern folgt auch postkoloniale Soziale Arbeit gerade nicht einem einheitlichen theoretischen Ansatz, weil sie von einer essentialistischen Theorie des Menschen oder normativen Idee der Gestaltung sozialer Beziehungen absieht und vielmehr als „Denken an den Grenzen“ (Hall 1995, S. 242), als Kritik von konkreten Machtverhältnissen in empirisch gegebener und damit unterschiedlicher Praxis zu verstehen ist. Daher wird Postkolonialismus inzwischen oft als Überbegriff für jedwede kritische Diagnose kultureller und wirtschaftlicher Machtverhältnisse mit globaler Orientierung genutzt. Trotz der unvermeidlichen Kontingenz und Asynchronität sowie der Widersprüche in den theoretischen Grundannahmen (vgl. etwa Chibber 2013 vs. Bahba 1994), ist die so suggerierte, alles Soziale umfassende Geltung sinnvoll: Der Makel der kolonialen Vergangenheit verbindet die gesamte Menschheit; ebenso wie der neoliberale Kapitalismus die erschreckend-faszinierende Fähigkeit besitzt, sogar die entferntesten Winkel zu unterwerfen (u. a. Chakrabarty 2000). Das postkoloniale Narrativ setzt also einer (implizit nationalen) Einheit des Sozialen eine (globale) 
Totalität der (differenzbedrohenden) Machtverhältnisse entgegen - was zugleich ein fruchtbares, nationale Ordnungen transzendierendes, diskursives Feld eröffnet hat.

\subsection{Konzeptionen des Pädagogischen}

Der Anspruch, ,,entfremdende“ Machtstrukturen ohne „,essentialistischen Gegenentwurf“ zu hinterfragen (Rosa 2016, S. 300), wie auch das Vorgehen, bestimmte Praktiken auszuwählen, welche dann etwa als Disziplinierungs- und Normierungsmechanismen analysiert werden können, trifft im Grunde auch für die kritische Theorie hier zu. Aus pädagogischer Sicht ist dies dort hinreichend, wo auf das Erwachen eines kritisch-reflexiven Bewusstseins um die eigenen (Denk-)Gewohnheiten oder herrschenden Zuschreibungen anderer hingearbeitet wird.

Für sozialpädagogische Kontexte wiederum scheint das nur bedingt sinnvoll. Zum einen gibt es jene Tradition, die auf die Erziehungsbedürftigkeit des Menschen durch Institutionen (Brezinka 1961) bzw. die Notwendigkeit von gesellschaftlicher Ordnung und Sozialisation verweist (Casale 2016) - wobei eben Theorien der Differenz den Bezugspunkt auf die strukturellen Machtverhältnisse zwischen Lebensweisen verlagern (statt gängiger Dichotomien wie etwa oben und unten). Zum anderen bedarf es in der Sozialen Arbeit Handlungsorientierungen, um in konkreten Lebenslagen beraten und biografische Prozesse moderieren, soziale Räume gestalten und Sozialpolitik entwerfen zu können. Hier ist weniger gemeint, dass die Soziale Arbeit oftmals von einer defizitär-therapeutischen Aufforderung zur Intervention ausgeht, sondern dass die Idee der Pädagogik auf die Gestaltung von Prozessen ausgelegt ist. Immer bedarf es nicht nur Argumente dagegen, sondern legitimer Gründe für eine Handlungsintention (auch für das Nicht-Handeln) oder eine richtungsweisende Idee der möglichen Veränderung(en) des Anderen. Darüber hinaus erklärt doch ein kritisch-reflexives Bewusstsein um (internalisierte) Machtverhältnisse nur sehr begrenzt, wann und wie ein anderes Verhalten innerhalb dieser oder entgegen diesen möglich ist. Als Praxiswissenschaft und als Profession zum Handeln verurteilt, zögert die Soziale Arbeit daher nicht, sich auf eine Reihe guter normativer Prinzipien (etwa Menschenrechte, Heteronormativität) zu berufen. Insofern besteht das Dilemma darin, dass in einer auf vielfältige Weise ungleichen Welt jeder Versuch des Gestaltens (im Hinblick auf das Eine) zugleich die überformende Wirkmächtigkeit von Kultur(en) reproduziert. Wie lassen sich also pädagogische Prozesse gestalten, die der Differenz gerecht werden?

Diese immer auch vereinnahmende Normativität bleibt aus postkolonialer Sicht problematisch (z.B. Said 1978), und es existieren verschiedene Debatten, anhand derer das tiefe Unbehagen im Versuch der Bearbeitung von Ungleichheit sichtbar wird. So wird etwa der Menschenrechtsansatz des UN-Systems (siehe Soziale Arbeit als Menschenrechtsprofession) vielerorts infrage gestellt: Obwohl gut gemeint und egalitär orientiert, wird damit doch ein eurozentrisches Werte- und Lebensmodell befördert, das die unendlich vielfältigen Ausdrucksformen von Kultur letztlich homogenisiert (z. B. Dunne et al. 2017, S. 35 f.). Auch löste Gayatri Spivaks Konzept des ,strategischen Essentialismus“ eine große Kontroverse innerhalb des Feldes aus, als sie behauptete, dass „Identität“ (und damit eine Vorstellung von Normalität) als zeitlich begrenzte Strategie für Aktivismus möglich sei, um so ein Zugehörigkeits- 
gefühl als Voraussetzung für kollektives Handeln zu schaffen (z. B. Ashcroft et al. 1998, S. 159). In ähnlicher Weise wurde Franz Fanons Klassiker „Schwarze Haut, weiße Masken“ (1952/1967) für die Darstellung kultureller Unterdrückungsmechanismen hoch verehrt, sein zweites Buch „Die Verdammten dieser Erde“ (1961/1963) von gleichgesinnten Akademiker*innen aber abgelehnt. In diesem Buch wendet sich Fanon ausdrücklich an die Befreiungskämpfe in Afrika und beschwört die Notwendigkeit von Nationalismus und die Bedeutung einer schwarzen Identität (Fanon 1961/1963, S. 32f.) - was er zuvor, in anderer Konstellation, in eindrücklicher Weise verteufelt hatte. Gerade in Lateinamerika wird unter der Chiffre dekolonial daher auch der bloßen Dekonstruktion von postkolonialen Machtverhältnissen immer ein Ansatz zur Seite gestellt, der, in dem so gewonnenen Freiraum, die Konstitution von Alternativen oder den Erhalt von präkolonialen Sozialformen betont (Muñoz Arce 2019).

Eine solche grundsätzliche Skepsis gegenüber normativen Zielen findet sich aber auch in den pädagogischen Ursprüngen Sozialer Arbeit - wurde dort aber anders aufgelöst, wohl, weil Handeln immer schon Ausgangspunkt der Theoriebildung war. Bereits Jean-Jacques Rousseau (1762/1998) prägte den Begriff der „Entfremdung“ durch die kulturellen „Ketten“ der Gesellschaft (Rousseau 1762/1998, S. 1), um demgegenüber eine pädagogische Utopie zu zeichnen, die (angeblich) nur der Subjektivität des*der Schüler*in folgt, bis diese*r stark genug ist, in die gesellschaftliche Auseinandersetzung um das Soziale einzutreten. „Emile“ ist somit eine seiner Antworten auf eben jenes Dilemma, das Rousseau im sogenannten „Zweiten Diskurs über die Ungleichheit unter den Menschen“ formuliert (Rousseau 1755/2012): Mit zunehmender Zivilisation bzw. fortschreitender Enkulturation schwinden die prinzipiell unbegrenzten Möglichkeiten des menschlichen Seins. Eine Denkfigur, die der Postkolonialismus aufgreift - verschärft durch die historische Erfahrung von tatsächlichen Ketten, zusammengepfercht im Bauch von Sklavenschiffen. Insbesondere Friedrich Schleiermacher (1826/2000) weist dann später der Pädagogik einen Weg aus diesem Dilemma, indem er Pädagogik als ethische Praktik auffasst, jenseits essentialistischer Annahmen über das Wesen des Menschen, und indem er jenseits kontingenter, historisch-interkultureller Ziele, das Gute formal bestimmt, eben ob kulturelle Reproduktion und subjektive Entfaltung in ,möglichster Zusammenstimmung“ stattfinden, anstatt sie im entfremdenden ,Widerspruch“ zu belassen (Schleiermacher 1826/2000, S. 34) - was nicht heißt, dass es aus feministischer oder postkolonialer Sicht nicht auch massive Einwände dagegen gäbe.

Jenseits einer individuell-therapeutisch-erziehenden Fallarbeit gibt es in der Sozialen Arbeit auch immer wieder prominente dezentrale Konzepte, etwa in der Rede von der Gestaltung des sozialen Raumes oder der „Lebensweltorientierung“ (Thiersch 1992), die jenseits von Erziehungssituationen Ermöglichungsräume nach ethischen Prinzipien schaffen wollen - und ohne gegenüber einzelnen Individuen konkrete Erwartungen an das Sein zu formulieren. Aus derselben Skepsis gegenüber einer essentialistischen wie überformenden Pädagogik heraus überdenkt auch Paulo Freire (1974) im brasilianischen Kontext die Erziehungssituation als Kern des Pädagogischen. Sein Weg führt aber nicht zu deren Auflösung, sondern er stärkt diese, indem er die Beziehung zwischen Lernenden und Lehrenden als egalitärreziproke Sozialbeziehung konzipiert, die dann innerhalb und gegenüber vermach- 
teten Lebenswelten wirkmächtig werden soll. Vor dem Hintergrund eines kritischen Bewusstseins um eine krass ungleiche Welt ist es für ihn aber zugleich nicht möglich, neutral zu sein, und jede professionelle Handlung (wie jedes Nicht-Handeln) muss sich beständig hinterfragen, ob sie bestehende Machtverhältnisse reproduziert oder nicht. Eine andere prägende Quelle Sozialer Arbeit in Brasilien war gewiss die Befreiungstheologie. Gekoppelt an eine Erfahrung krasser Landflucht und prekarisierender Urbanisierung, kommt eine Deutung zur Geltung, nach welcher der Verlust der ländlichen Gemeinschaften einerseits und eine perspektivlose, urbane Armut andererseits zur inneren Destabilisierung geführt haben und kompensiert werden müssen. Die Praxis Sozialer Arbeit, etwa mit Obdachlosen, Landlosen und Müllsammler*innen, war daher ab den 1970er Jahren dadurch geprägt, in Stadtteilprojekten lokale, kollektive Gemeinschaften aufzubauen bzw. im ländlichen Raum der Destabilisierung solcher Gemeinschaften entgegenzuwirken. Zusammenhalt und soziales Miteinander, als Rahmen für den Aufbau einer inneren Ordnung und deren Stabilisierung in tagtäglich herausfordernden Zusammenhängen, werden so zu einer lokalen Antwort, ohne eine einende Totalität vorauszusetzen.

\subsection{Die prozesshafte Gestaltung des Sozialen}

In der hiesigen Sozialpädagogik, die durch das Denken in Prozessen, aber auch durch den Bildungsbegriff beeinflusst ist, gibt es die Idee, dass der Aufbau einer ,inneren Lebensordnung“ nur in wechselseitig prozesshafter Bezugnahme auf Institutionen (im weiteren Sinne) und die umgebende soziale Ordnung sowie in konfliktreicher Auseinandersetzung damit möglich ist (Winkler 2000, S. $211 \mathrm{f}$.). Gerade weil dies unter „Bedingungen der Veränderung“ besonders gut gelingt, sind soziale Bewegungen herausragende Orte Sozialer Arbeit, in Abgrenzung zu den stark institutionalisierten hiesigen Ansätzen (Bunk 2017); eine Idee, die in Lateinamerika sehr ausgeprägt ist. Der gemeinsame Kampf um Anerkennung bzw. die konflikthafte Auseinandersetzung mit herrschenden Institutionen, die Aufarbeitung kolonialer Geschichte oder die einende Bezugnahme auf gegenwärtige (strukturelle) Machtmechanismen (national wie global) sind zudem konstitutive Prozesse für kollektive Subjekte (Caldart 2004), die so zugleich individuelle Bildungsprozesse ermöglichen, moderieren und dynamisieren (Bunk 2016, 2018).

Tatsächlich wäre es aber eine zu einfache Zuschreibung, würde man Theorien der Differenz per se vorhalten, bei einer antagonistischen Kritik des Bestehenden stehen zu bleiben. Besonders prominent betont Gayatri Spivak (2010), dass die Lösung kolonialer Machtverhältnisse nicht im Austausch der Positionen in diesem Gefüge liege (also nicht die Beherrschten sich zu neuen Herrscher*innen aufschwingen), sondern eben in der Überwindung von Machtverhältnissen, was sich als permanente Aufgabe erweist, die zunehmend alle Bereiche zu durchdringen habe. An dieser Stelle, um diesen Prozess der Überwindung zu umschreiben, führt Spivak doch das Soziale ein. Nämlich ein ,kollektives Bemühen“ zum ,Wohle des ganzen Globus, nicht nur der vierten Welt", das ,langsam und in beide Richtungen aufmerksam ist" - und findet als ethischen Maßstab dieses „Lernprozesses“ dann zu dem Begriff „Liebe“, die zu dem kollektiven Bemühen hinzukommen muss und dessen Beschaffenheit charakterisiert (Spivak 2010, S. 65). Bei allen Unterschieden erinnert diese Denkfigur wieder 
sehr an Überlegungen etwa Hartmut Rosas (2019), die eingangs als Gegenentwurf zu einer antagonistischen Sozialontologie angedeutet wurden - und die im Grunde bildungstheoretisch fundiert sind, weil sie die präfigurative Bedeutung einer prozessorientierten gesellschaftlichen Aushandlung für die individuelle „Anverwandlung“ von Welt und den Aufbau von Selbstverhältnissen betonen. Daher fordert H. Rosa einen ,demokratischen Resonanzraum, in dessen Zentrum nicht der Kampf, sondern die Gestaltung steht und als dessen regulative Idee nicht die Hegemonie, sondern das Gemeinwohl dient, als Denk- und Erfahrungsmöglichkeit“ (Rosa 2019, S. 242).

In Brasilien - oder Lateinamerika, also dort, wo das Soziale krass ungleich ist und nicht von allen den Anderen Teilhabe zuerkannt wird - verlagert sich insofern die konstitutive Bedeutung sozialer Aushandlungsprozesse auf eine andere Ebene. Im Vordergrund Sozialer Arbeit stehen kollektive Subjekte - ob nun soziale Bewegungen oder traditionelle lokale Gemeinschaften, ob urbane Stadtteile oder alternative ökonomische Projekte -, die es aufzubauen, zu stabilisieren oder zu stärken gilt, weil sie der Rahmen sind, in welchem das Soziale, als Gemeinschaft oder Gemeinwohl, angesichts der exkludierenden Polarisierung dort gedacht und vor allem erfahren werden kann (womit nicht ein utopisch-harmonisches Miteinander dieser Kollektive, ohne Makel, Missbrauch und Gewalt gemeint ist). Als solche distinkt wahrnehmbaren und dadurch der teilhabenden Gestaltung zugänglichen (Beziehungs-)Zusammenhänge treten diese kollektiven Subjekte dann ein, in ein größeres Ringen um die Konstitution des Sozialen an sich - ohne die eigene Erfahrungsmöglichkeit eines (niederschwelligeren) Sozialen beständig aufs Spiel zu setzen. Dabei vertreten sie durchaus eine einende, übergeordnete Idee des zunehmend demokratischen Miteinanders, bzw. eines sozialen Zusammenhaltes, welcher in seiner inklusiven Anlage über eine territoriale Einheit hinausgeht, aber nicht als gegeben vorausgesetzt wird. Sie stehen damit im Gegensatz zu anderen wirtschaftlichen, religiösen und nationalkonservativen Projekten, für welche die Auswirkungen des eigenen Tuns und Agierens auf (gegenwärtige oder künftige) Andere schlicht belanglos sind und die ihre (exklusive) soziale Entwicklung gerade trotz der Anderen (und möglichst unbehelligt von diesen) vorantreiben. Insofern haben sich alle diese Ansätze Sozialer Arbeit (im weiteren Sinne) der guten Bewältigung des Sozialen verschrieben, ausgehend von einer Perspektive der Differenz. Daher zielen sie auf die Stärkung subalterner Ordnungen, sozialer Bewegungen, lokaler Wissenskulturen oder alternativer (tradierter) Lebensweisen im urbanen wie im ländlichen Raum - gerade weil sie individuelle Bildungsprozesse im Ringen um eine alle umfassende Idee des Sozialen im Blick haben.

Danksagung Die Idee zu diesem Blickpunkt entstand während eines Feodor Lynen-Forschungsaufenthaltes (2017-2018), gefördert durch die Alexander von Humboldt-Stiftung. Die Umsetzungen des Heftes wurde durch das Dekanat Sozial- und Kulturwissenschaften und den Bereich Pädagogik des Jugendalters an der Justus-Liebig-Universität Gießen unterstützt.

Funding Open Access funding enabled and organized by Projekt DEAL.

Open Access Dieser Artikel wird unter der Creative Commons Namensnennung 4.0 International Lizenz veröffentlicht, welche die Nutzung, Vervielfältigung, Bearbeitung, Verbreitung und Wiedergabe in jeglichem Medium und Format erlaubt, sofern Sie den/die ursprünglichen Autor(en) und die Quelle ordnungsgemäß nennen, einen Link zur Creative Commons Lizenz beifügen und angeben, ob Änderungen vorgenommen wurden. 
Die in diesem Artikel enthaltenen Bilder und sonstiges Drittmaterial unterliegen ebenfalls der genannten Creative Commons Lizenz, sofern sich aus der Abbildungslegende nichts anderes ergibt. Sofern das betreffende Material nicht unter der genannten Creative Commons Lizenz steht und die betreffende Handlung nicht nach gesetzlichen Vorschriften erlaubt ist, ist für die oben aufgeführten Weiterverwendungen des Materials die Einwilligung des jeweiligen Rechteinhabers einzuholen.

Weitere Details zur Lizenz entnehmen Sie bitte der Lizenzinformation auf http://creativecommons.org/ licenses/by/4.0/deed.de.

\section{Literatur}

Alvaredo, F., Chancel, L., Piketty, T., Saez, E., \& Zucman, G. (2018). Die weltweite Ungleichheit. Der World Inequality Report 2018. München: C.H. Beck.

Ashcroft, B., Griffiths, G., \& Tiffin, H. (1998). Post-colonial studies. The key concepts. London: Routledge.

Bahba, H. K. (1994). The location of culture. London: Routledge.

Bäumer, G. (1929). Die historischen und sozialen Voraussetzungen der Sozialpädagogik und die Entwicklung ihrer Theorie. In H. Nohl \& L. Pallat (Hrsg.), Handbuch der Pädagogik, Bd. 5. Langensalza: Beltz.

Becker, R., \& Streiß, T. (2018). Sich in die Nesseln setzen. Zum Verhältnis von Antirassismus und Feminismus. In K. Linkerhand (Hrsg.), Feministisch streiten. Texte zu Vernunft und Leidenschaft unter Frauen. Berlin: Querverlag.

Biller, M. (2014). Letzte Ausfahrt Uckermark. Die Zeit 9/2014. 20.02.2014. https://www.zeit.de/2014/09/ deutsche-gegenwartsliteratur-maxim-biller. Zugegriffen: 20. Okt. 2020.

Biller, M. (2019). Sind Sie auch ein Linksrechtsdeutscher? Welt. 16.02.2019. https://www.welt.de/kultur/ literarischewelt/article188904661/Maxim-Biller-Sind-Sie-auch-ein-Linksrechtsdeutscher.html. Zugegriffen: 20. Okt. 2020.

Bresser-Pereira, L.C. (2018). Capitalismo financeiro-rentista. Estudos Avançados, 32(92), 17-29. https:// doi.org/10.5935/0103-4014.20180003.

Brezinka, W. (1961). Der erziehungsbedürftige Mensch und die Institutionen. In W. Brezinka (Hrsg.), Weltweite Erziehung (S. 11-35). Freiburg: Herder.

Bunk, B. (2016). Soziale Bewegungen und Bildungstheorie. Überlegungen zu Bildungsprozessen in sozialen Bewegung(en). Forschungsjournal Soziale Bewegungen, 29(4), 30-38.

Bunk, B. (2017). Zur Differenz von Sozialer Arbeit und sozialer Bewegung. In D. Franke-Meyer \& C. Kuhlmann (Hrsg.), Soziale Arbeit und Soziale Bewegung (S. 265-280). Wiesbaden: VS.

Bunk, B. (2018). Bildung und Soziale Bewegung. Die Movimento dos Sem Terra und das World Social Forum als Räume für Bildungsprozesse. Paderborn: Schöningh.

Caldart, R. (2004). Pedagogia do Movimento Sem Terra. São Paulo: Expressão Popular.

Casale, R. (2016). Krise der Repräsentation. Zur Sittlichkeit des Staates und Autorität des Vaters. In R. Casale, H.-C. Koller \& N. Ricken (Hrsg.), Das Pädagogische und das Politische. Zu einem Topos der Erziehungs- und Bildungsphilosophie. Paderborn: Schöningh.

Chakrabarty, D. (2000). Provincializing Europe: postcolonial thought and historical difference. Princeton: Princeton University Press.

Chibber, V. (2013). Postcolonial theory and the specter of capital. A provocative intellectual assault on the Subalternists' foundational work. New York: Verso Books.

Connell, R. (2006). Northern Theory. The political geography of general social theory. Theory and Society, 35(2), 237-264.

Della, T. (2020). „Rassismus braucht keine Intention“ (Interview von M. Barišić). Süddeutsche Zeitung. 09.06.2020. https://www.sueddeutsche.de/panorama/black-lives-matter-george-floyd-rassismus-alllives-matter-1.4932258. Zugegriffen: 20. Okt. 2020.

Dunne, M., Durrani, N., Fincham, K., \& Crossouard, B. (2017). Troubling muslim youth identities. Nation, religion, gender. Basingstoke: Palgrave Macmillan.

El-Mafaalani, A. (2018). Das Integrationsparadox. Köln: Kiepenheuer \& Witsch.

Estevam, D. (2020). Emergência da extrema direita e ameaças à democracia no Brasil. ritimo. 01.01.2020. https://www.ritimo.org/Emergencia-da-extrema-direita-e-ameacas-a-democracia-no-Brasil. Zugegriffen: 20. Okt. 2020.

Evers, A., \& Nowotny, H. (1987). Über den Umgang mit Unsicherheit. Die Entdeckung der Gestaltbarkeit von Gesellschaft. Frankfurt a. M.: Suhrkamp. 
Fanon, F. (1963). The wretched of the earth. New York: Grove Weidenfeld.

Fanon, F. (1967). Black skin, white masks. New York: Grove Press.

Flaßpöhler, S. (2019). „Hören Sie auf, Sie beleidigen uns!“ Von moralischem Totalitarismus (Interview von P. Unfried \& H. Welzer). Taz. Futurzwei. 12.06.2019. https://taz.de/Von-moralischemTotalitarismus/!168884/. Zugegriffen: 20. Okt. 2020.

Freire, P. (1974). Pedagogia do Oprimido. Rio de Janeiro: Paz e Terra.

Ghiringhelli de Azevedo, R. (2020). A Mitomania. Sul21. 25.05.2020. https://www.sul21.com.br/ opiniaopublica/2020/05/a-mitomania-por-rodrigo-ghiringhelli-de-azevedo/. Zugegriffen: 20. Okt. 2020.

Gore, T., Alestig, M., \& Ratcliff, A. (2020). Confronting carbon inequality. Putting climate justice at the heart of the COVID-19 recovery. (Oxfam Media Briefing 21.09.2020). https://www.oxfam.de/system/ files/documents/20200921-confronting-carbon-inequality.pdf. Zugegriffen: 20. Okt. 2020.

Habermas, J. (1992). Staatsbürgerschaft und nationale Identität. In J. Habermas (Hrsg.), Faktizität und Geltung: Beiträge zur Diskurstheorie des Rechts und des demokratischen Rechtsstaats (S. 632-660). Frankfurt a. M.: Suhrkamp.

Habermas, J. (1994). Struggles for recognition in the democratic constitutional state. In A. Gutmann (Hrsg.), Multiculturalism: examining the politics of recognition (S. 107-148). Princeton: Princeton University Press.

Hall, S. (1995). When was the ,post-colonial'? Thinking at the limit. In I. Chambers \& L. Curti (Hrsg.), Postcolonial question: common skies, divided horizons (S. 242-260). London: Routledge.

Honneth, A. (2011). Das Recht der Freiheit: Grundriss einer demokratischen Sittlichkeit. Berlin: Suhrkamp.

INCRA (Instituto Nacional de Colonização e Reforma Agrária, 1996). Atlas Fundiario do Brasil. Brasília: INCRA.

Kessl, F. (2011). Demokratietheoretische Vergewisserung in der Sozialen Arbeit. In B. Lösch \& A. Thimmel (Hrsg.), Kritische politische Bildung. Ein Handbuch, Bd. 1085 (S. 129-141). Bonn: bpb (Bundeszentrale für politische Bildung).

Lessenich, S. (2008). Die Neuerfindung des Sozialen. Der Sozialstaat im flexiblen Kapitalismus. Bielefeld: transcript.

Lessenich, S. (2016). Neben uns die Sintflut. Die Externalisierungsgesellschaft und ihr Preis. München, Berlin: Hanser.

Leyendecker, P. (2018). „Ich möchte Herrn Gauland nicht zuhören“ (Interview von P. Schwarz). Christ \& Welt, Beilage in Die Zeit 40/2018. https://www.zeit.de/2018/40/evangelischer-kirchentag-afdausladung-hans-leyendecker. Zugegriffen: 20. Okt. 2020.

Marchart, O. (2010). Die politische Differenz. Frankfurt a. M.: Suhrkamp.

Marchart, O. (2019). Der feindliche Zwilling. Herbert Marcuses Theorie der Politik aus postfundamentalistischer Perspektive. In U. Bohmann \& P. Sörensen (Hrsg.), Kritische Theorie der Politik (S. 143-178). Frankfurt a. M.: Suhrkamp.

Mecheril, P., Thomas-Olalde, O., Melter, C., Arens, S., \& Romaner, E. (Hrsg.). (2013). Migrationsforschung als Kritik? Spielräume kritischer Migrationsforschung. Wiesbaden: Springer VS.

Mignolo, W.D. (2007). Introduction: coloniality of power and de-colonial thinking. Cultural Studies, 21, $155-167$.

Moore, J. (Hrsg.). (2016). Anthropocene or capitalocene? Nature, history, and the crisis of capitalism. Oakland: PM Press.

Muñoz Arce, G. (2019). Latin American social work and the struggles against professional Imperialism. In R. Lutz, T. Kleibel, N. Noyoo, B. Bunk, B. Seepamore \& A. Dittmann (Hrsg.), The Routledge handbook of postcolonial social work (S. 163-173). London: Routledge.

Newmark, C. (2017). Identitätspolitik. Alle sind betroffen. Die Zeit. 19.09.2017. https://www.zeit.de/ kultur/2017-09/identitaetspolitik-bundestagswahl-queere-people-of-color-frauen. Zugegriffen: 20. Okt. 2020.

Oliveira, L.L. (1990). A questão nacional na primeira República. São Paulo: Brasiliense.

Otto, H. U., Thiersch, H., Treptow, R., \& Ziegler, H. (Hrsg.). (2018). Handbuch Soziale Arbeit. Grundlagen der Sozialarbeit und Sozialpädagogik. München: Ernst Reinhardt.

Prengel, A. (2007). Grundlagen und Probleme der Pädagogik der Vielfalt. In G. Krell, B. Riedmüller, B. Sieben \& D. Vinz (Hrsg.), Diversity Studies. Grundlagen und disziplinäre Ansätze (S. 49-67). Frankfurt a. M., New York: Campus.

Quijano, A. (2000). Colonialidad del poder, eurocentrismo y Am é rica Latina. In E. Lander (Hrsg.), La colonialidad del saber: eurocentrismo y ciencias sociales. Perspectivas latino americanas (S. 201-246). Buenos Aires: CLACSO. 
Rosa, H. (2016). Resonanz. Eine Soziologie der Weltbeziehung. Frankfurt a. M.: Suhrkamp.

Rosa, H. (2019). Der Irrtum der antagonistischen Sozialontologie. Zur kritischen Theorie demokratischer Resonanz. In U. Bohmann \& P. Sörensen (Hrsg.), Kritische Theorie der Politik (S. 209-242). Frankfurt a. M.: Suhrkamp.

Rousseau, J. (1998). Emil oder über die Erziehung. Paderborn: Schöningh.

Rousseau, J. (2012). Discours sur les sciences et les arts. Abhandlung über die Wissenschaften und die Künste. Stuttgart: UTB. deutsch-frz. Ausgabe, hrsg. von D. Butz-Striebel \& B. Durand

Said, E. (1978). Orientalism. New York: Pantheon.

Sandkötter, S. (1999). Modernisierungsforschung in Brasilien. Campus Forschung, Bd. 796. Frankfurt a. M.: Campus.

Schleiermacher, F. (2000). Grundzüge der Erziehungskunst (Vorlesungen 1826). In M. Winkler \& J. Brachmann (Hrsg.), Texte zur Pädagogik. Kommentierte Studienausgabe Bd. 2. Frankfurt a. M.: Suhrkamp.

Sobottka, E. A. (2006). The responsibility of governing and the changes in the workers' party of Brazil. International Journal of Action Research, 2(1), 54-57.

Sobottka, E. A. (2019). Constelações pós-nacionais e a questão da integração social. Caderno CRH, 32(85), 47-62. https://doi.org/10.9771/ccrh.v32i85.27683.

de Sousa Santos, B. (1994). Pela mão de Alice. o social e o político na pós-modernidade. Porto: Afrontamento.

Spivak, G. C. (2010). Kultur. In J. Reuter \& P.-I. Villa (Hrsg.), Postkoloniale Soziologie (S. 47-68). Bielefeld: transcript. Englisches Original in dies. (1999), A Critique of Postcolonial Reason: Toward a History of the Vanishing Present. Cambridge, MA: Harvard University Press.

Staub-Bernasconi, S. (2007). Soziale Arbeit als Handlungswissenschaft. Systemische Grundlagen und professionelle Praxis - Ein Lehrbuch. Bern: Haupt/UTB.

Taylor, C. (1994). The Politics of Recognition. In A. Gutmann (Hrsg.), Multiculturalism: examining the politics of recognition (S. 25-73). Princeton: Princeton University Press.

Taylor, C. (2002). Wieviel Gemeinschaft braucht die Demokratie? Aufsätze zur politischen Philosophie. Frankfurt a. M.: Suhrkamp.

Thiersch, H. (1992). Lebensweltorientierte soziale Arbeit. Aufgaben der Praxis im sozialen Wandel. Weinheim: Juventa.

Wenzel, O. (2020). 1000 Serpentinen Angst (Roman). Frankfurt a. M.: S. Fischer.

Winkler, M. (2000). Anton Makarenko. In M. Buchka, R. Grimm \& F. Klein (Hrsg.), Lebensbilder bedeutender Heilpädagoginnen und Heilpädagogen im 20. Jahrhundert. München: Ernst Reinhardt.

Winkler, W. (2006). Kleine Skizze einer revidierten Theorie der Sozialpädagogik. In T. Badawia, H. Luckas \& H. Müller (Hrsg.), Das Soziale gestalten. Über Mögliches und Unmögliches der Sozialpädagogik (S. 55-80). Wiesbaden: Springer VS.

Žižek, S. (2019). Sex and the Failed Absolute. In S. Žižek (Hrsg.), Death of Truth (S. 103-106). London: Bloomsbury Academic. 